University of Wollongong

Research Online

Faculty of Engineering and Information

Faculty of Engineering and Information

Sciences - Papers: Part A

Sciences

$1-1-2014$

\title{
Panoptes: Calibration of a dosimetry system for eye brachytherapy
}

\author{
Michael Weaver \\ University of Wollongong, mweaver@uow.edu.au \\ Marco Petasecca \\ University of Wollongong, marcop@uow.edu.au \\ Dean Cutajar \\ University of Wollongong, deanc@uow.edu.au \\ Michael L. F Lerch \\ University of Wollongong, mlerch@uow.edu.au \\ Gianluigi De Geronimo \\ Brookhaven National Laboratory
}

See next page for additional authors

Follow this and additional works at: https://ro.uow.edu.au/eispapers

Part of the Engineering Commons, and the Science and Technology Studies Commons

Research Online is the open access institutional repository for the University of Wollongong. For further information contact the UOW Library: research-pubs@uow.edu.au 


\title{
Panoptes: Calibration of a dosimetry system for eye brachytherapy
}

\begin{abstract}
Intraocular cancer is a serious threat to the lives of those that suffer from it. Dosimetry for eye brachytherapy presents a significant challenge due to the inherently steep dose gradients that are needed to treat such small tumours in close proximity to sensitive normal structures. This issue is addressed by providing much needed quality assurance to eye brachytherapy, a novel volumetric dosimetry system, called Panoptes was developed. This study focuses on the preliminary characterisation and calibration of the system. Using ion beam facilities, the custom, pixelated silicon detector of Panoptes was shown to have good charge collection uniformity and a well defined sensitive volume. Flat-field calibration was conducted on the device using a $250 \mathrm{kVp}$ orthovoltage beam. Finally, the detector and phantom were simulated with Monte Carlo in Geant4, to create water equivalent dose correction factors for each pixel across a range of angles.
\end{abstract}

\section{Keywords}

brachytherapy, system, panoptes, calibration, eye, dosimetry

Disciplines

Engineering | Science and Technology Studies

\section{Publication Details}

Weaver, M., Petasecca, M., Cutajar, D. L., Lerch, M. L. F., De Geronimo, G., Pinelli, D. A., Cullen, A. J., Prokopovich, D. A., Perevertaylo, V. L. \& Rosenfeld, A. B. (2014). Panoptes: Calibration of a dosimetry system for eye brachytherapy. Radiation Measurements, 71 310-314.

\section{Authors}

Michael Weaver, Marco Petasecca, Dean Cutajar, Michael L. F Lerch, Gianluigi De Geronimo, Donald Pinelli, Ashley Cullen, Dale A. Prokopovich, Vladimir Perevertaylo, and Anatoly B. Rosenfeld 


\title{
PANOPTES: Calibration of a dosimetry system for eye brachytherapy
}

\author{
Pixelated, Automated Nuclear Ophthalmic Plaque Treatment Evaluation System
}

\author{
M. Weaver ${ }^{a, *}$, M. Petasecca ${ }^{\mathrm{a}}$, D. L. Cutajar ${ }^{\mathrm{a}}$, M. L. F. Lerch $^{\mathrm{a}}$, G. De Geronimo ${ }^{\mathrm{b}}$, D. A. Pinelli ${ }^{\mathrm{b}}$, A. J. Cullen ${ }^{\mathrm{c}}$, D. A. \\ Prokopovich $^{\mathrm{d}}$, V. L. Perevertaylo ${ }^{\mathrm{e}}$, A. B. Rosenfeld ${ }^{\mathrm{a}, * *}$ \\ ${ }^{a}$ Centre for Medical Radiation Physics, University of Wollongong, NSW, Australia \\ ${ }^{b}$ Brookhaven National Laboratory, Upton, NY 11973, USA \\ ${ }^{c}$ Illawarra Cancer Care Centre, Wollongong, NSW, Australia \\ ${ }^{d}$ Australian Nuclear Science and Technology Organisation, Lucas Heights, NSW, Australia \\ e $S P A-B I T$, Kiev, Ukraine 04136
}

\begin{abstract}
Intraocular cancer is a serious threat to the lives of those that suffer from it. Dosimetry for eye brachytherapy presents a significant challenge due to the inherently steep dose gradients that are needed to treat such small tumours in close proximity to sensitive normal structures. This issue is addressed by providing much needed quality assurance to eye brachytherapy, a novel volumetric dosimetry system, called PANOPTES was developed. This study focuses on the preliminary characterisation and calibration of the system. Using ion beam facilities, the custom, pixelated silicon detector of PANOPTES was shown to have good charge collection uniformity and a well defined sensitive volume. Flatfield calibration was conducted on the device using a $250 \mathrm{kVp}$ orthovoltage beam. Finally, the detector and phantom were simulated with Monte Carlo in Geant4, to create water equivalent dose correction factors for each pixel across a range of angles.
\end{abstract}

Keywords: eye plaque, brachytherapy, dosimetry, HERMES, IBICC

\section{Introduction}

Although not as prevalent as some tumours, intraocular cancers present a significant risk to sufferers $[1,4$, $5,7]$. Uveal melanoma is the most commonly occurring of these and can be treated with a number of modalities. Brachytherapy is the most widely used radiotherapy treatment for posterior uveal melanoma [7]. It involves suturing a radioactive source, in the form of a small dish shaped device called a plaque, directly over the tumour for the treatment period. Once this duration has expired, it is removed again. Typical radionuclides used include ${ }^{103} \mathrm{Pd}$ or ${ }^{125} \mathrm{I}$ as small rice-like seeds that fit into an insert or ${ }^{106} \mathrm{Ru}$ as a uniform coating. The system presented in this study focuses on ${ }^{125} \mathrm{I}$ plaques, though limitations in the measurement of other radioisotopes are not yet apparent.

Despite improvements over the years, uncertainties and limitations still remain in eye brachytherapy including: loss of vision and other iatrogenic side effects; inability to treat large tumours; and failure to eliminate the threat of tumour. Due to the presence of surrounding vital structures and the small tumour sizes involved, averaging around

\footnotetext{
* Corresponding author

** Principal corresponding author

Email addresses: mweaver@uow.edu.au (M. Weaver), anatoly@uow.edu.au (A. B. Rosenfeld)
}

$12 \mathrm{~mm}$ diameter by $6 \mathrm{~mm}$ thick, dose gradients must be very steep and are innately difficult to measure.

Currently, no treatment planning system (TPS) is recommended by either the American Association of Physicists in Medicine (AAPM) or American Brachytherapy Society $(\mathrm{ABS})$ as a primary dose calculation tool because none are certified by either the Food and Drug Authorities (FDA) or Conformité Européenne (c $\epsilon$ ). The Task Group $43 \mathrm{U} 1$ report formalism [6] is used as the industry standard for dose calculation but assumes infinite water surrounds and cannot account for material heterogeneities. The extensive TG-129 Report[2] provides an excellent summary and recommendations of such issues regarding the current status of dosimetry for eye plaques. From this, it is suggested that treatment planning can be improved with a quality assurance system that offers fast, direct dose verification of eye plaques that: measures the dose close to the plaque surface; has fast 3D dose mapping; can display dose near critical structures and; accounts for dose in many heterogeneous materials thus acting to complement current TPSs. This study details a system, called the PANOPTES, which provides at method of physically measuring dose distributions in eye plaques. 


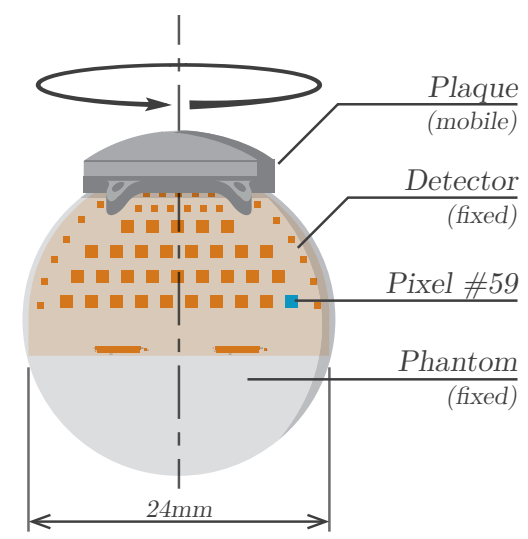

Figure 1: Volumetric dosimetry concept based on superimposing multiple measurements taken at a variety of plaque-detector angles.

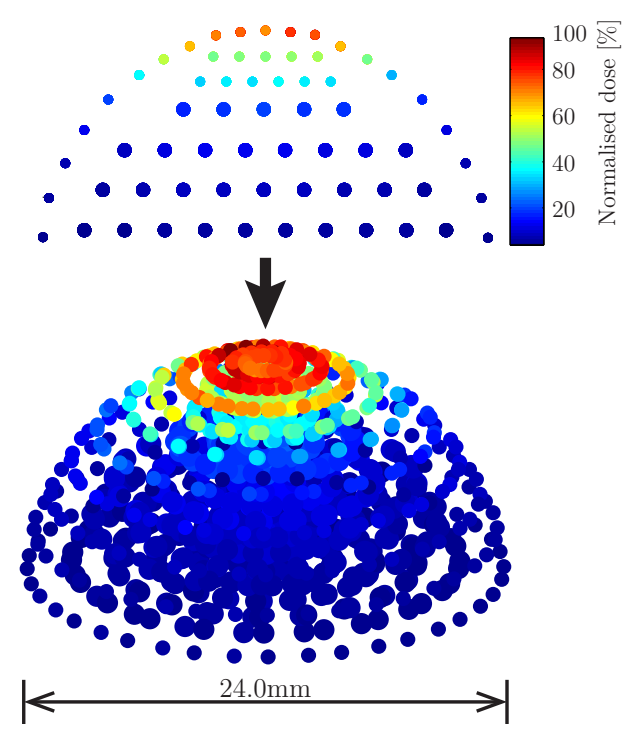

Figure 2: Single planar measurements (top) are made at multiple plaque-detector angles using the PANOPTES and superposed to create a dose volume (bottom).

\section{Methods and Materials}

Volumetric dosimetry for eye brachytherapy can be achieved through the super-positioning of multiple planar dose measurements. This study has lead to the development of a volumetric dosimeter which operates by rotating the plaque, using a stepper motor, about the axis of a $24 \mathrm{~mm}$ wide semicircular detector, see Figure 1. The detector shape allows for dose measurement from less than $1 \mathrm{~mm}$ from the inner surface of the plaque to the centre of the eye. This gives good dosimetric coverage of the volume of the eye surrounding the tumour from which other points of interest can be extrapolated. Each measurement of the detector produces a planar semicircular dose map which can then be reconstructed, through the superposition of multiple angles, into a single hemispherical dose volume, such as the example shown in Figure 2.

Following the success of early results with the Medipix

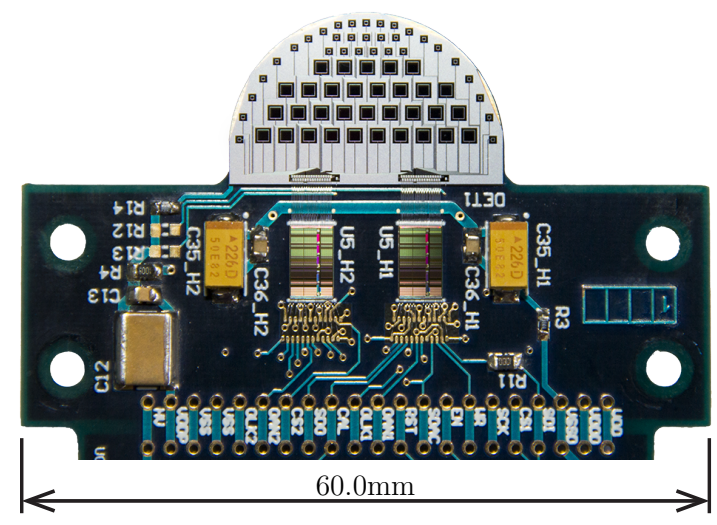

Figure 3: Photograph of detector mounted on readout board and bonded to two HERMES4[3], mixed-signal front-end ASICs.

detectors [8, 9], this study has developed the PANOPTES, the pixelated, automated nuclear ophthalmic plaque treatment evaluation system. It features a custom designed and in-house fabricated detector that consists of an array of 64 silicon $p$ - $i$ - $n$ diodes on a single semicircular cut $300 \mu \mathrm{m}$ wafer, aimed at providing dosimetry close to the plaque surface.

The detector was wire bonded to two HERMES4[3], low noise, mixed-signal front-end application specific integrated circuits (ASICs) with 24-bit counters and three separate energy windows. The ASIC was interfaced to a graphical user interface for readout via a field-programmable gate array (FPGA). Figure 3 shows the detector with the two HERMES ASICs bonded below.

In order to use the device for dosimetry, it must first be characterised and calibrated. Characterisation was carried out, in part, using ion-beam induced charge collection (IBICC) at the ANTARES Accelerator at ANSTOd During this process, ions of $5.5 \mathrm{MeV}$ helium and $11.75 \mathrm{MeV}$ beryllium were focused to approximately one micrometer and raster scanned across the surface of the detector. The collected charge was readout using an AMPTEK A250 preamplifier where median energy maps and spectra were subsequently produced. A number of measurements were taken adjusting several variables including; bias voltage, scan size, ion type, gain and connected pixel. The data were calibrated using a series known pulse inputs.

Preliminary detector calibration was conducted using two methods: flat-field $\gamma$-ray and Monte Carlo simulation. Flat-field calibration of two detector samples was carried out at the $\mathrm{ICCC}^{\mathrm{c}}$, using a Gulmay D3300, orthovoltage machine. The sample, without any phantom, was placed at a distance of three meters from the source in a free air environment. The HERMES4 was configured with a peaking time of $0.5 \mu \mathrm{s}$, gain of $0.75 \mathrm{~V} / \mathrm{fC}$ and energy thresholds set above background noise. The detector was fully depleted at a reverse bias voltage of $50 \mathrm{~V}$ (based on IBICC results). Using an energy of $250 \mathrm{kVp}, 100$ acquisitions of $0.1 \mathrm{~s}$ were taken and integrated for a total time of $10 \mathrm{~s}$. These values were then normalised against the maximum 
value to produce the calibration factors for each pixel.

Energy correction was calculated using Geant4 version 9.5 patch 01 with Livermore low energy physics models for Monte Carlo simulation. This involved determining the silicon to water dose ratio coefficients for each pixel in the silicon detector averaged over $360^{\circ}$. The simulation included modelling the full PANOPTES detector, with sensitive volumes corresponding to the pixels placed inside a poly(methyl methacrylate) (PMMA) eye phantom. A $15 \mathrm{~mm}$ ROPES eye plaque containing ten Oncura model $6711^{125}$ I seeds was located at the apex of the eye phantom. Dose was measured in two eye phantom configurations; one with the semicircular silicon pixelated detector sandwiched between two PMMA hemispheres in air; and the other with the silicon, PMMA and air, all replaced by water. These configurations were used to represent the phantom apparatus and treatment conditions for calculation of the silicon to water dose ratio coefficients.

The angular response of each pixel was measured by varying the plaque axial rotation from $0^{\circ}$ to $180^{\circ}$ in $15^{\circ}$ increments. Due to the symmetry of the model, full the $360^{\circ}$ could be extrapolated. These factors were then averaged and compared to determine the per average pixel dose correction factors and their deviations.

\section{Results and Discussion}

The TG-43U1 method of calculating dose has been shown to be significantly different from physical measurement and simulation, as summaries in The Task Group 129 Report[2]. The differences have were shown to be up to $30 \%$ off axis and could be accounted for with the implementation of a dosimetry system such as the PANOPTES, improving the accuracy of dose treatment planning.

In preparation of the PANOPTES detector for absolute dosimetry its pixel volumes was examined in a flat-field, as described above, and correction factors were determined for sensitivity and energy response.

\section{1. $I B I C C$}

The median energy maps and the corresponding energy spectrum for a single, large pixel (\#59, located most bottom-right of detector, see Figure 1) are displayed in Figures 4 and 5, respectively. For this measurement the $5.5 \mathrm{MeV} \mathrm{He}^{2+}$ ion was used with a $50 \mathrm{~V}$ bias and a scan magnification of 6 . The energy map shows uniform charge collection across the detector surface and a well defined sensitive volume. The definition of this sensitive volume is critical for accurate, relative dosimetry. Similar results were observed in other pixels measured, both small and large in size.

Charge sharing between pixels was tested by comparing floating to biased pixel neighbours and showed there was no significant difference. This is important in accurate dosimetry to ensure that dose is correctly mapped to its corresponding area.

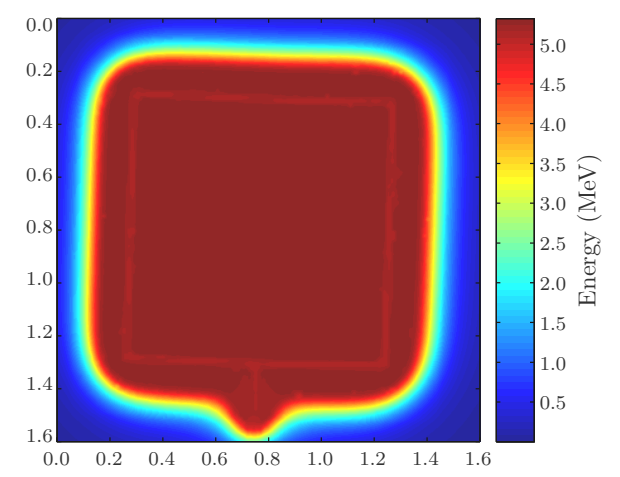

Figure 4: Ion-beam induced charge collection median energy map (\#59, most bottom-right of large pixels) showing well defined sensitive volume and aluminium contact pad. Image axes are given in millimetres.

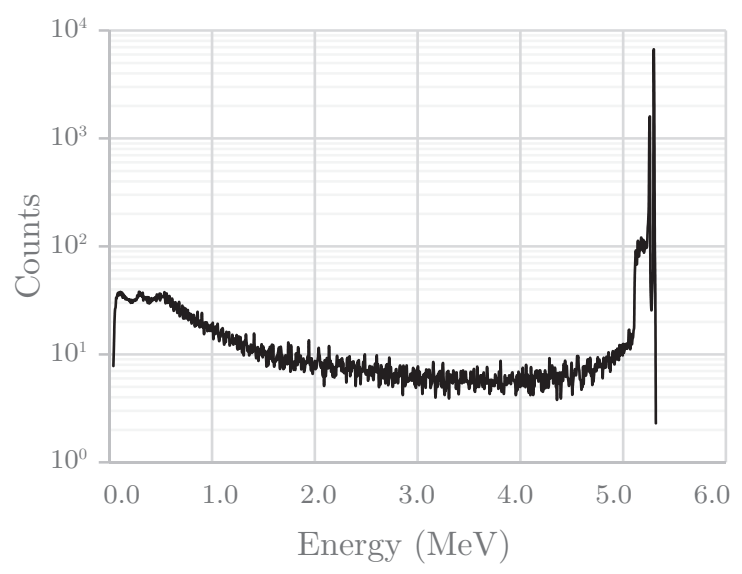

Figure 5: Energy spectrum corresponding to the median energy map in Figure 4, showing full charge collection at incident particle energy.

A comparison of the spectra taken from several median energy maps showed that full charge collection occurs at the incident particle energy for a bias voltage of $50 \mathrm{~V}$. This voltage was thus selected as the operational voltage of the detector. A small shoulder to the left of this peak is contributed by the $\mathrm{Al}$ metallisation surrounding the perimeter of the $p+$ implanted region (visible in Figure 4) and will have negligible effect on ${ }^{125} \mathrm{I}$ and ${ }^{106} \mathrm{Ru}$ dosimetry.

\subsection{Flat-field calibration}

The flat-field calibration factors for the individual pixels are displayed in Figure 6. There are two distinct groups depicted, showing the small pixels at the top and the large pixels at the bottom, with mean values of $8.40(\sigma=0.63)$ and $1.10(\sigma=0.06)$ relative to maximum pixel response, respectively. The small pixels have an equivalently higher calibration factor than the large pixels $(\sim 7.5$ times) due to the lower amount of energy they receive as a result of having a smaller sensitive volume. Good uniformity across large pixels was observed, while small pixels showed a greater deviation, due, in part, to great variations in track length. The longer tracks contribute a higher capacitance relative to the capacitance of the small pixels, which 


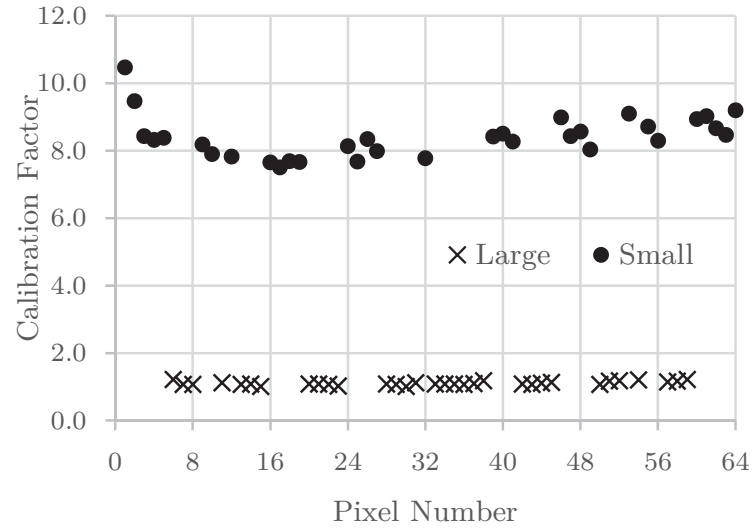

Figure 6: Flat-field calibration factors of all pixels, showing the effect of pixel geometry on measurable sensitivity.

leads to reduced response. During measurements, the response from each PANOPTES detector will be divided by the flat-field calibration factors to normalise the responses in conjunction with the Monte Carlo generated energy correction factors.

\subsection{Monte Carlo Energy Correction}

The water equivalent dose correction factors calculated for each pixel as the average of all angles are displayed in Figure 7. The error bars for each data point represent one standard deviation of the discrepancy across all angles. With these correction factors applied, simulated PANoptes data, calculated as silicon pixels in PMMA and converted to dose in water per unit area, predicted treatment dose rates to within $2 \%$ for most pixels and up to $9.5 \%$ for pixels furthest from the plaque. Whilst these values predominantly lie within current treatment uncertainties, further improvement could be achieved by implementing angle specific correction factors instead of averaged across all angles, the accuracy of which would need to be measured experimentally. The pixels with the lowest deviation across the range of angles are those located in the top-central region of the detector, directly over the most likely tumour location, providing excellent intratumour accuracy. Angular dependence must be thoroughly considered to ensure that the system responds as expected, especially when dealing with customised plaque designs.

The Monte Carlo correction factors obtained for each pixel will be used in conjunction with the above flat-field calibration to create individual correction factors for each pixel in PANOPTES measurements.

\section{Conclusion}

The steep dose gradients that must be used for treating intraocular tumours cause great challenges in dosimetry measurements for the treatment. Until a TPS is approved by the AAPM, the TG-43U1 is often used as a pri-

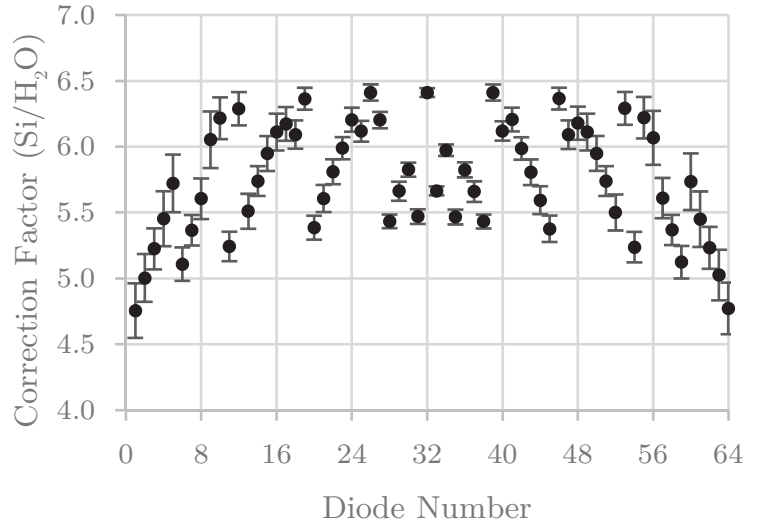

Figure 7: Simulated $\mathrm{Si}: \mathrm{H}_{2} \mathrm{O}$ dose ratio correction factors for each pixel averaged across all angles. Error bars indicate one standard deviation.

mary dose calculation tool, though it does not account for material heterogeneities. The TG-129 Report summarises several studies showing that the presence of the plaque has a significant effect of dose in comparison to homogenous water[2] and should be considered in dose calculations. The current state of eye brachytherapy treatment verification is lagging behind technological feasibility.

Characterisation of the novel pixelated, semicircular detector using ion beam facilities has shown that the sensitive volume of the detector is well defined, charge collection is uniform and charge sharing between pixels is not present. These qualities are important for accurate, relative dosimetry. The detector's operational voltage was determined $(50 \mathrm{~V})$ through observing the spectra for full charge collection.

Calibration of the detector accounting for individual pixel sensitivity was conducted using flat-field $\gamma$-rays from an $250 \mathrm{kV}$ p orthovoltage machine. In addition to this, Monte Carlo simulations using Geant 4 were used to calculate dose to water equivalence correction factors for each pixel, averaged for all angles from $0^{\circ}$ to $360^{\circ}$ in $15^{\circ}$ increments. The energy correction factors fall to within $2 \%$ for most pixels while the maximum deviation across all pixels and angles is $9.5 \%$.

Providing a reliable volumetric dosimetry tool, such as PANOPTES, for pretreatment dose verification would be an excellent complement to current TPSs for eye brachytherapy. Such a tool would not only give medical physicists more confidence in their treatments, but also allow them to develop patient specific, customised plaques with the increasing popularity of $3 \mathrm{D}$ printing.

\section{Acknowledgements}

The authors would like to acknowledge support from the Medipix Collaboration, M. Zaider (Memorial SloanKettering Cancer Center), M. Carolan (Illawarra Cancer Care Centre), M. Conway (Sydney Eye Hospital), T. Kron 
(Peter MacCallum Cancer Centre) and an NHMRC Grant: ID 573428 .

[1] Bedikian, A.Y., 2006. Metastatic uveal melanoma therapy: current options. International ophthalmology clinics 46, 151-66.

[2] Chiu-Tsao, S.T., Astrahan, M.a., Finger, P.T., Followill, D.S., Meigooni, A.S., Melhus, C.S., Mourtada, F., Napolitano, M.E., Nath, R., Rivard, M.J., Rogers, D.W.O., Thomson, R.M., 2012. Dosimetry of (125)I and (103)Pd COMS eye plaques for intraocular tumors: report of Task Group 129 by the AAPM and ABS. Medical physics 39, 6161-84. doi:10.1118/1.4749933.

[3] De Geronimo, G., O'Connor, P., Beuttenmuller, R., Li, Z., Kuczewski, A., Siddons, D., 2003. Development of a high-rate high-resolution detector for EXAFS experiments. IEEE Transactions on Nuclear Science 50, 885-891. doi:10.1109/TNS. 2003. 814540.

[4] Kujala, E., Ma, T., 2003. Very Long-Term Prognosis of Patients with Malignant Uveal Melanoma. Investigative Ophthalmology \& Visual Science 44, 4651-4659. doi:10.1167/iovs.03-0538.

[5] Lutz, J.M., Cree, I.a., Foss, a.J., 1999. Risk factors for intraocular melanoma and occupational exposure. The British journal of ophthalmology 83, 1190-3.

[6] Rivard, M.J., Coursey, B.M., DeWerd, L.a., Hanson, W.F., Saiful Huq, M., Ibbott, G.S., Mitch, M.G., Nath, R., Williamson, J.F. 2004. Update of AAPM Task Group No. 43 Report: A revised AAPM protocol for brachytherapy dose calculations. Medical Physics 31, 633. doi:10.1118/1.1646040.

[7] Shields, C.L., Shields, J.A., 2009. Ocular melanoma: relatively rare but requiring respect. Clinics in dermatology 27, 122-33. doi:10.1016/j.clindermatol.2008.09.010.

[8] Weaver, M., Green, J., Petasecca, M., Lerch, M., Cutajar, D. Franklin, D., Jakubek, J., Carolan, M., Conway, M., Pospisil, S., Kron, T., Metcalfe, P., Zaider, M., Rosenfeld, A., 2011a. Threedimensional dosimetry imaging of I-125 plaque for eye cancer treatment. Nuclear Instruments and Methods in Physics Research Section A: Accelerators, Spectrometers, Detectors and Associated Equipment 633, S276-S278. doi:10.1016/j.nima. 2010. 06.187.

[9] Weaver, M., Petasecca, M., Lerch, M., Cutajar, D., JakÅŕbek, J., Pospíšil, S., Rosenfeld, A., 2011b. Dosimetry verification in eye brachytherapy using silicon pixelated detectors. Radiation Measurements 46, 2010-2013. doi:10.1016/j.radmeas.2011.10. 008. 